\title{
Effectiveness of neuromuscular electrical stimulation for interstitial cystitis: a protocol of systematic review and meta-analysis
}

\section{Da-yin Chen}

First Affiliated Hospital of Jiamusi University

\section{Ying-xue Guo}

Jiamusi University

\section{Long-xin Dong}

First Affiliated Hospital of Jiamusi University

Wen-jie He

First Affiliated Hospital of Jiamusi University

\section{Hui-feng Cao}

First Affiliated Hospital of Jiamusi University

\section{Ping Wang}

Criminal Technology Division of Jiamusi Public Security Bureau

Cai-fang Yue ( $\nabla$ yezhaonei2@126.com )

First Affiliated Hospital of Jiamusi University

\section{Protocol}

Keywords: Interstitial cystitis, neuromuscular electrical stimulation, effectiveness, safety

Posted Date: May 14th, 2020

DOl: https://doi.org/10.21203/rs.3.rs-27664/v1

License: (c) (i) This work is licensed under a Creative Commons Attribution 4.0 International License. Read Full License 


\section{Abstract}

Background This study will examine the effectiveness and safety of neuromuscular electrical stimulation (NMES) for the treatment of patients with interstitial cystitis (IC).

Methods We will retrieve the following electronic databases from their commencements to the March 1 , 2020 to discover all related potential studies: MEDLINE, EMBASE, Cochrane Library, Web of Science, CINAHL, China National Knowledge Infrastructure, Chinese Biomedical Literature Database, Chinese Scientific Journal Database, and WANFANG Database. Randomized controlled trials (RCTs) related to the NMES for the treatment of patients with IC will be included, regardless publication status and language. Literature selection, data collection, and study quality assessment will be independently performed by two authors. The extracted data will be expressed as risk ratio and $95 \%$ confidence intervals (Cls) for dichotomous data, and mean difference or standard mean difference and $95 \%$ Cls for continuous data. RevMan V.5.3 software will be employed for statistical analysis.

Discussion This study will summarize current high quality RCTs to appraise the effectiveness and safety of NMES for the treatment of patients with IC. The findings of this study will provide helpful evidence to determine whether NMES is an effective treatment for patients with IC or not. Systematic review registration PROSPERO CRD42020170495.

\section{Background}

Interstitial cystitis (IC) is a chronic, progressive debilitating bladder disorder [1-3], which is characterized by intermittent flares of frequency, urgent voiding, and pelvic pain [4-6]. It is estimated that the prevalence rate of IC ranges from $0.3 \%$ to $2 \%$ according to clinical diagnosis around the world [7-8]. The etiology of IC remains poorly understood [9]. Despite several therapies are reported to manage IC, none of them can effectively treat this condition [10-11]. Thus, it is very urgent to find potential candidates.

Fortunately, neuromuscular electrical stimulation (NMES) is recommended as a potential candidate and a variety of studies reported that NMES can be used to treat IC [12-26], which offers us new data for conducting this systematic review. Accordingly, this present study is designed to critically synthesize the most recent published data to appraise the effectiveness and safety of NMES for the treatment of patients with IC.

\section{Methods}

\section{Study registration}


This protocol has been registered on PROSPERO (CRD42020170495), and it is reported in accordance with the Preferred Reporting Items for Systematic Reviews and Meta-Analysis (PRISRMA) Protocol statement guidelines [27-28].

\section{Study eligibility criteria}

\section{Types of studies}

Without limitations on language and publication status, this study will include randomized controlled trials (RCTs) that explore the effectiveness and safety of NMES for the treatment of patients with IC.

\section{Types of participants}

All patients who were diagnosed as IC will be included, regardless gender, race, age, economic status, duration and severity of IC.

\section{Types of interventions}

\section{Experimental interventions}

All patients in the experimental group were treated with NMES only. Any treatments combined with NMES will be excluded.

\section{Control interventions}

All patients in the control group were treated with any interventions, such as oral medication, moxibustion, and physical therapy. However, any management combined with NMES will be excluded.

\section{Type of outcome measurements}

Primary outcomes are pain intensity (as measured by Visual Analog Scale or other pain scales), and improvement of overall symptoms (as assessed by patient-reported global response assessment or other tools). 
Secondary outcomes are urinary frequency episodes, quality of life (as checked by 36-Item Short Form Survey or other questionnaires), and adverse events.

\section{Search strategy and data management}

\section{Search strategy}

The electronic databases will be comprehensively retrieved from their commencements to the March 1 , 2020 to identify all related potential studies: MEDLINE, EMBASE, Cochrane Library, Web of Science, CINAHL, China National Knowledge Infrastructure, Chinese Biomedical Literature Database, Chinese Scientific Journal Database, and WANFANG Database. There are no limitations to publication language and status. A search strategy for MEDLINE has been established (table 1). Identical search strategies will be applied to all other electronic databases.

We will also search relevant conference abstracts, clinical trial registries for ongoing trials, and reference lists of all related reviews.

\section{Study selection}

Two authors will independently import all citations into EndNote X9 to eliminate duplicated ones. Titles/abstracts of all potential records will be screened to remove any irrelevant studies. If necessary, we will obtain and read full-text of remaining literatures according to the eligibility criteria. All excluded studies will be noted and summarized with reasons. Any differences will be worked out with the help of another author and a consensus will be made. The results of study selection will be summarized in a PRISRMA flow diagram.

\section{Data extraction and management}

Two authors will independently extract data by a standardized data form developed specifically for this study. Any confusion will be cleared up with the help of another author and a final conclusion will be made. The information includes study general information (e.g. title, first author, year of publication), participant characteristics (age, gender), diagnostic criteria, inclusion and exclusion criteria, study design, sample size, interventions, outcomes, results, findings, adverse events, and funding information.

\section{Dealing with missing data}


Any insufficient or missing data will be requested from primary authors. If they can not provide those data, we will perform data analysis based on the available data, and we will discuss its affects on the study findings.

\section{Study quality assessment}

The Cochrane Collaboration's tool will be utilized to assess study quality of included trials by two independent authors. Each study will be identified at seven aspects, and each one is graded as 3 levels: low, unclear, and high risk of bias. Divergences will be arbitrated with the help of another author.

\section{Statistical analysis}

\section{Data synthesis}

RevMan 5.3 software will be utilized for statistical analysis. Risk ratio with $95 \%$ confidence intervals (Cls) will be used to measure the treatment effect for dichotomous outcome data. Mean difference or standardized mean difference and $95 \%$ Cls will be suggested to measure the treatment effect for continuous outcome data. Statistical heterogeneity will be examined by $R$ test. $R \leq 50 \%$ suggests homogeneity, and we will use a fixed-effect model. If possible, we will conduct a meta-analysis when sufficient trials are included. $R>50 \%$ indicates considerable heterogeneity, and we will place a randomeffect model. In addition, we will perform subgroup analysis to check sources of obvious heterogeneity. If there is still remarkable heterogeneity after subgroup analysis, a meta-analysis will not be carried out. However, we will report outcome results using a narrative synthesis.

\section{Subgroup analysis}

If studies are adequate, we will perform a subgroup analysis based on the different types of interventions, controls, and outcome measurements.

\section{Sensitivity analysis}

Whenever it is appropriate, we will undertake a sensitivity analysis to test stability of study findings by removing low quality studies, or small sample studies.

\section{Reporting bias}


We will adopt a funnel plot and Egger's regression test to investigate reporting bias if 10 or more trials are included [29-30].

\section{Grading the quality of evidence}

Two authors will independently appraise quality of evidence for each outcome by Grading of Recommendations Assessment, Development and Evaluation, which grades quality of evidence as 4 levels: high, moderate, low, and very low [31]. Any uncertainty will be solved with the help of an independent arbitrator.

\section{Dissemination}

This study will be disseminated through a peer-reviewed journal or a conference meeting.

\section{Discussion}

Some studies have shown that NMES may benefit patients with IC [12-26]. However, the results are still not consistent, and so far no systematic review of NMES in treating IC has been identified. Thus, this study intends to present the merged data and to carry out a systematic review of NMES for IC in order to supply high-quality evidence. Its results may provide reference and recommendation for clinician and scientific searchers.

\section{Abbreviations}

NMES, neuromuscular electrical stimulation; IC, interstitial cystitis; RCTs, randomized controlled trials; Cls, confidence intervals.

\section{Declarations}

Ethics approval and consent to participate: Not applicable.

Consent for publication: Not applicable.

Availability of data and material: Data sharing is not applicable to this article as no datasets were generated or analyzed during the current protocol. 
Competing interests: Not applicable.

Funding: This work has supported by the Scientific Research Project of Heilongjiang Provincial Department of Health (2019-329). The supporter will just provide financial support and will not participate this study.

Authors' contributions: DYC, YXG and CFY conceived the study. LXD and WJH contributed with the clinical background and expertise. HFC, PW and CFY contributed with the analytical plan and the bias assessment approach. DYC, YXG, LXD, WJH, HFC, and CFY performed the literature search plan and drafted the protocol. All authors revised the protocol and approved the final version. CFY supervised the study

Acknowledgements: Not applicable.

Authors' information: Da-yin Chen, dayinc0284@outlook.com

Ying-xue Guo, noathesfqttk@outlook.com

Long-xin Dong, mcwheemevdlx@outlook.com

Wen-jie He, tollaslppfri@outlook.com

Hui-feng Cao, stesteyxomy@outlook.com

Ping Wang, heautiezimxnr@outlook.com

Cai-fang Yue, yezhaonei2@126.com

\section{References}

1. Barr S. Diagnosis and management of interstitial cystitis. Obstet Gynecol Clin North Am 2014; 41(3):397-407.

2. McLennan MT. Interstitial cystitis: epidemiology, pathophysiology, and clinical presentation. Obstet Gynecol Clin North Am 2014; 41(3):385-95. 
3. Vij M, Srikrishna S, Cardozo L. Interstitial cystitis: diagnosis and management. Eur J Obstet Gynecol Reprod Biol 2012; 161(1):1-7.

4. Meyer D, Gregorin J, Schmid HP. Diagnosis and treatment of interstitial cystitis. Praxis (Bern 1994) 2011; 100(4):221-7.

5. van de Merwe JP, Nordling J, Bouchelouche P, et al. Diagnostic criteria, classification, and nomenclature for painful bladder syndrome/interstitial cystitis: an ESSIC proposal. Eur Urol 2008; 53(1):60-7.

6. Hanno PM, Erickson D, Moldwin R, Faraday MM, American Urological Association. Diagnosis and treatment of interstitial cystitis/bladder pain syndrome: AUA guideline amendment. J Urol 2015; 193(5):1545-53.

7. Nickel JC, Teichman JM, Gregoire M, Clark J, Downey J. Prevalence, diagnosis, characterization, and treatment of prostatitis, interstitial cystitis, and epididymitis in outpatient urological practice: the Canadian PIE Study. Urology 2005; 66: 935-40.

8. Bogart LM, Suttorp MJ, Elliott MN, Clemens JQ, Berry SH. Prevalence and correlates of sexual dysfunction among women with bladder pain syndrome/interstitial cystitis. Urology 2011; 77(3):57680.

9. Birder LA. Pathophysiology of interstitial cystitis. Int J Urol 2019; 26 (Suppl 1):12-15.

10. Ha T, Xu JH. Interstitial cystitis intravesical therapy. Transl Androl Urol 2017; 6(Suppl 2):S171-9.

11. Giusto LL, Zahner PM, Shoskes DA. An evaluation of the pharmacotherapy for interstitial cystitis. Expert Opin Pharmacother 2018; 19(10):1097-108.

12. Gonzalez EJ, Grill. Sensory pudendal nerve stimulation increases bladder capacity through sympathetic mechanisms in cyclophosphamide-induced cystitis rats. Neurourol Urodyn 2019; 38(1):135-43.

13. Peters KM, Killinger KA, Gilleran JP, Bartley J, Wolfert C, Boura JA. Predictors of reoperation after sacral neuromodulation: A single institution evaluation of over 400 patients. Neurourol Urodyn 2017; 36(2):354-9.

14. Geirsson G, Wang YH, Lindström S, Fall M. Traditional acupuncture and electrical stimulation of the posterior tibial nerve. A trial in chronic interstitial cystitis. Scand J Urol Nephrol 1993; 27(1):67-70.

15. Fall M, Carlsson CA, Erlandson BE. Electrical stimulation in interstitial cystitis. J Urol 1980; 123(2):192-5.

16. Parsons $M$, De Jong P, Radziszewski $D$, et al. Analysis of long-term pelvic floor electrostimulation therapy for interstitial crititis. European Urology Supplements 2006; 5(2):193.

17. Bruce F. Initial Results of Pelvic Floor Electrostimulation for Symptom Relief in Interstitial Cystitis [online]. Australian and New Zealand Continence Journal 2005; 11(2):38-40.

18. Zhao JM, Nordling J. Posterior tibial nerve stimulation in patients with intractable interstitial cystitis[J]. BJU Int 2004; 94(1):101-4. 
19. Comiter CV. Sacral Neuromodulation for the Symptomatic Treatment of Refractory Interstitial Cystitis: A Prospective Study. J Urol 2003; 169(4):1369-73.

20. Buffington CA, Teng B, Somogyi GT. Norepinephrine content and adrenoceptor function in the bladder of cats with feline interstitial cystitis. J Urol 2002; 167(4):1876-80.

21. Zermann $D H$, Weirich $T$, Wunderlich $H$, Reichelt $O$, Schubert J. Sacral nerve stimulation for pain relief in interstitial cystitis. Urol Int 2000; 65(2):120-1.

22. Fall $M$, Lindström S.Transcutaneous electrical nerve stimulation in classic and nonulcer interstitial cystitis. Urol Clin North Am 1994; 21(1):131-9.

23. Fall M. Conservative management of chronic interstitial cystitis: transcutaneous electrical nerve stimulation and transurethral resection. J Urol 1985; 133(5):774-8.

24. Zhang W, Wang DW, Cao XM, Hu CY, Guo H, Gao JP. Observation of the therapeutic effect of percutaneous electrical stimulation on refractory lower urinary tract symptoms in elderly women. Chinese Journal of Geriatrics 2019; 9:1024-7.

25. Li XL. Observation of the clinical effect of transcutaneous electrical stimulation and biofeedback in the treatment of interstitial cystitis / bladder pain syndrome. Chinese Journal of Practical Medicine 2019; 14 (11):35-6.

26. Yi QT, Lv JW, Jiang C, Lv TT, Fu QB. Biofeedback and electrical stimulation in the treatment of nonulcerative interstitial cystitis / bladder pain syndrome. Shanghai Medical 2016; 37(16):6-8.

27. Shamseer L, Moher D, Clarke M, et al. PRISMA-P Group. Preferred reporting items for systematic review and meta-analysis protocols (PRISMA-P) 2015: elaboration and explanation. BMJ 2015; 349: g7647.

28. Moher D, Shamseer $L$, Clarke $M$, et al.Preferred reporting items for systematic review and metaanalysis protocols (PRISMA-P) 2015 statement. Syst Rev 2015;4:1.

29. Sutton AJ, Duval SJ, Tweedie RL, et al. Empirical assessment of effect of publication bias on metaanalyses. BMJ 2000; 320:1574-1577.

30. Egger M, Davey Smith G, Schneider M, et al. Bias in meta-analysis detected by a simple, graphical test. BMJ 1997; 315:629-634.

31. Guyatt GH, Oxman AD, Vist GE, et al. GRADE: an emerging consensus on rating quality of evidence and strength of recommendations. BMJ 2008; 336:924-6.

\section{Tables}

Table 1 Search strategy used in MEDLINE database 


\begin{tabular}{ll}
\hline Number & Search terms \\
\hline 1 & interstitial cystitis \\
2 & in-tur-STISH-ul sis-TIE-tis \\
3 & painful bladder syndrome \\
4 & bladder pain \\
5 & painful bladder syndrom \\
6 & urinary urgency \\
7 & Or 1-6 \\
8 & electrical stimulation \\
9 & neuromuscular \\
10 & NMES \\
11 & neuroprosthetics \\
12 & electrical therapy \\
13 & electrical treatment \\
14 & Or 8-13 \\
15 & random \\
16 & randomly \\
17 & allocation \\
18 & blind \\
19 & placebo \\
20 & sham \\
21 & controlled trial \\
22 & clinical trial \\
23 & Or 15-22 \\
24 & 7 and 14 and 23 \\
\hline & \\
\hline
\end{tabular}

\section{Supplementary Files}

This is a list of supplementary files associated with this preprint. Click to download.

- PRISMAPchecklist.doc 